PROCEEDINGS OF THE

AMERICAN MATHEMATICAL SOCIETY

Volume 137, Number 11, November 2009, Pages 3919-3922

S 0002-9939(09)09966-3

Article electronically published on June 18, 2009

\title{
BETTI NUMBERS AND INJECTIVITY RADII
}

\author{
MARC CULLER AND PETER B. SHALEN
}

(Communicated by Daniel Ruberman)

Dedicated to José María Montesinos on the occasion of his 65th birthday

\begin{abstract}
We give lower bounds on the maximal injectivity radius for a closed hyperbolic 3-manifold with first Betti number 2 under some additional topological hypotheses.
\end{abstract}

The theme of this paper is the connection between topological properties of a closed orientable hyperbolic 3-manifold $M$ and the maximal injectivity radius of $M$. In 4] we showed that if the first Betti number of $M$ is at least 3, then the maximal injectivity radius of $M$ is at least $\log 3$. By contrast, the best known lower bound for the maximal injectivity radius of $M$ with no topological restriction on $M$ is the lower bound of $\operatorname{arcsinh}\left(\frac{1}{4}\right)=0.24746 \ldots$ due to Przeworski [7]. One of the results of this paper, Corollary 4, gives a lower bound of 0.32798 for the case where the first Betti number of $M$ is 2 and $M$ does not contain a "fibroid" (see below). Our main result, Theorem 3, is somewhat stronger than this.

The proofs of our results combine a result due to Andrew Przeworski [7] with results from [5] and [6].

The results of [5] and 6] were motivated by applications to the study of hyperbolic volume, and these applications were superseded by the results of [2]. The applications presented in the present paper do not seem to be accessible by other methods.

As in [5], we define a book of I-bundles to be a compact, connected, orientable topological 3-manifold (with boundary) $W$ which has the form $W=\mathcal{P} \cup \mathcal{B}$, where

- $\mathcal{P}$ is an $I$-bundle over a non-empty compact 2-manifold-with-boundary,

- each component of $\mathcal{B}$ is homeomorphic to $D^{2} \times S^{1}$,

- the set $\mathcal{A}=\mathcal{P} \cap \mathcal{B}$ is the vertical boundary of the $I$-bundle $\mathcal{P}$, and

- each component of $\mathcal{A}$ is an annulus in $\partial \mathcal{B}$ which is homotopically non-trivial in $\mathcal{B}$.

(Note that this terminology differs slightly from that of [1, where it is the triple $(W, \mathcal{B}, \mathcal{P})$ that is called a book of $I$-bundles.)

As in [5], we define a fibroid in a closed, connected, orientable 3-manifold $M$ to be a connected incompressible surface $F$ with the property that each component of the compact manifold obtained by cutting $M$ along $F$ is a book of $I$-bundles.

Received by the editors February 12, 2009.

2000 Mathematics Subject Classification. Primary 57M50; Secondary 57N10.

This work was partially supported by NSF grants DMS-0504975 and DMS-0608567.

(C)2009 American Mathematical Society Reverts to public domain 28 years from publication 
Note that in defining a fibroid to be connected, we are following the convention of [5] rather than that of [6].

We define a function $R(x)$ for $0<x<\log 3$ by

$$
R(x)=\frac{1}{2} \operatorname{arccosh}\left(\frac{e^{2 x}+2 e^{x}+5}{\left(\cosh \frac{x}{2}\right)\left(e^{x}-1\right)\left(e^{x}+3\right)}\right) .
$$

As in [3, Section 10], we define a function $V(x)$ for $0<x<\log 3$ by

$$
V(x)=\pi x \sinh ^{2} R(x)=\frac{\pi x}{e^{x}-1}\left(\frac{e^{2 x}+2 e^{x}+5}{2\left(e^{x}+3\right) \cosh (x / 2)}\right)-\frac{\pi x}{2} .
$$

Thus, in a closed hyperbolic 3-manifold, if a geodesic of length $\ell$ is the core of an embedded tube of radius $R(\ell)$, then this tube has volume $V(\ell)$.

The following result is implicit in [6], but we will supply a proof for the sake of comprehensibility.

Proposition 1. Let $M$ be a closed hyperbolic 3-manifold. Suppose that there is an infinite subset $\mathcal{N}$ of $H_{2}(M ; \mathbb{Z})$ such that every element of $\mathcal{N}$ is represented by some connected, incompressible surface which is not a fibroid. Let $\lambda$ be a positive number less than $\log 3$. Then either the maximal injectivity radius of $M$ is at least $\lambda / 2$ or $M$ contains a closed geodesic $C$ of length at most $\lambda$ such that the maximal tube about $C$ has radius at least $R(\lambda)$ and volume at least $V(\lambda)$, where $R(\lambda)$ and $V(\lambda)$ are defined by (11) and (2).

Proof. The hypothesis implies in particular that $H_{2}(M ; \mathbb{Z})$ has infinitely many primitive elements, and so the first Betti number $\beta_{1}(M)$ is at least 2. If $\beta_{1}(M) \geq 3$, then according to [4, Corollary 10.4], the maximal injectivity radius is at least $\frac{1}{2} \log 3>\lambda / 2$. We may therefore assume that $\beta_{1}(M)=2$. Hence the quotient of $H_{1}(M, \mathbb{Z})$ by its torsion subgroup is a free abelian group $L$ of rank 2 . We let $h: \pi_{1}(M) \rightarrow L$ denote the natural homomorphism.

We distinguish two cases. First consider the case in which $M$ contains a nontrivial closed geodesic $C$ of some length $\ell<\lambda$ such that the conjugacy class represented by $C$ is contained in the kernel of $h$. Let $T$ denote the maximal embedded tube about $C$. According to [3, Corollary 10.5] we have $\operatorname{Vol} T \geq V(\lambda)$. If $\rho$ denotes the radius of $T$, this gives

$$
\pi \ell \sinh ^{2} \rho=\operatorname{Vol} T \geq V(\lambda)=\pi \lambda \sinh ^{2} R(\lambda)>\pi \ell \sinh ^{2} R(\lambda),
$$

and hence $\rho>R(\lambda)$.

Thus the second alternative of the proposition holds in this case.

We now turn to the case in which no non-trivial closed geodesic of length $<\lambda$ represents a conjugacy class contained in the kernel of $h$. Since $M$ is closed, there are only a finite number $n \geq 0$ of conjugacy classes in $\pi_{1}(M)$ that are represented by closed geodesics of length $<\lambda$. Let $\gamma_{1}, \ldots, \gamma_{n}$ be elements belonging to these $n$ conjugacy classes. Then $\bar{\gamma}_{i}=h\left(\gamma_{i}\right)$ is a non-trivial element of $L$ for $i=1, \ldots, n$. Since $L$ is a free abelian group of rank 2 , there exists, for each $i \in\{1, \ldots, n\}$, a homomorphism $\phi_{i}$ of $L$ onto $\mathbb{Z}$ such that $\phi_{i}\left(\bar{\gamma}_{i}\right)=0$. Because $\bar{\gamma}_{i} \neq 0$, the epimorphism $\phi_{i}$ is unique up to sign.

The epimorphism $\phi_{i} \circ h: \pi_{1}(M) \rightarrow \mathbb{Z}$ corresponds to a primitive element of $H^{1}(M ; \mathbb{Z})$, whose Poincaré dual in $H_{2}(M ; \mathbb{Z})$ we shall denote by $c_{i}$. Since the set $\mathcal{N} \subset H_{2}(M ; \mathbb{Z})$ given by the hypothesis of the theorem is infinite, there is an element $c$ of $\mathcal{N}$ which is distinct from $\pm c_{i}$ for $i=1, \ldots, n$. Since $c \in \mathcal{N}$ it follows 
from the hypothesis that there is a connected incompressible surface $S \subset M$ which represents the homology class $c$ and is not a fibroid.

We now apply Theorem A of [5], which asserts that if $S$ is a connected nonfibroid incompressible surface in a closed, orientable hyperbolic 3-manifold $M$, and if $\lambda$ is any positive number, then either (i) $M$ contains a non-trivial closed geodesic of length $<\lambda$ which is homotopic in $M$ to a closed curve in $M-S$ or (ii) $M$ contains a hyperbolic ball of radius $\lambda / 2$. In the present situation, with $\lambda$ chosen as above, we claim that alternative (i) of the conclusion of Theorem A of [5] cannot hold.

Indeed, suppose that $C$ is a non-trivial closed geodesic of length $<\lambda$ with the properties stated in (i). Since $C$ has length $<\lambda$, the conjugacy class represented by $C$ contains $\gamma_{i}$ for some $i \in\{1, \ldots, n\}$. Since $C$ is homotopic to a closed curve in $M-S$, it follows that the image of $\gamma_{i}$ in $H_{1}(M ; \mathbb{Z})$ has homological intersection number 0 with $c$. Thus if $\psi: \pi_{1}(M) \rightarrow \mathbb{Z}$ is the homomorphism corresponding to the Poincaré dual of $c$, we have $\psi\left(\gamma_{i}\right)=0$. Now since $L$ is the quotient of $H_{1}(M)$ by its torsion subgroup, $\psi$ factors as $\phi \circ h$, where $\phi$ is some homomorphism from $L$ to $\mathbb{Z}$. Since $c$ is primitive, $\psi$ is surjective, and hence so is $\phi$. But we have $\phi\left(\bar{\gamma}_{i}\right)=\psi\left(\gamma_{i}\right)=0$. In view of the uniqueness that we observed above for $\phi_{i}$, it follows that $\phi= \pm \phi_{i}$, so that $\psi= \pm \phi_{i} \circ h$ and hence $c= \pm c_{i}$. This contradicts our choice of $c$.

Hence (ii) must hold. This means that the maximal injectivity radius of $M$ is at least $\lambda / 2$. Thus the first alternative of the proposition holds in this case.

Proposition 2. Let $M$ be a closed hyperbolic 3-manifold. Suppose that there is an infinite subset $\mathcal{N}$ of $H_{2}(M ; \mathbb{Z})$ such that every element of $\mathcal{N}$ is represented by some connected, incompressible surface which is not a fibroid. Then the maximal injectivity radius of $M$ exceeds 0.32798 .

Proof. We set $\lambda=2 \times 0.32798=0.65596$.

According to Proposition 1, either the maximal injectivity radius of $M$ is at least $\lambda / 2$ - so that the conclusion of the theorem holds - or $M$ contains a closed geodesic $C$ of length at most $\lambda$ such that the maximal tube about $C$ has volume at least $V(\lambda)$, where $V(\lambda)$ is defined by (2). In the latter case, if $R$ denotes the radius of $T$, we have

$$
R \geq R(\lambda)=0.806787 \ldots
$$

Now according to [7, Proposition 4.1], the maximal injectivity radius of $M$ is bounded below by

$$
\operatorname{arcsinh}\left(\frac{\tanh R}{2}\right)>\operatorname{arcsinh}\left(\frac{\tanh 0.806787}{2}\right)>0.32799 .
$$

This gives the conclusion of the theorem in this case.

Theorem 3. Let $M$ be a closed hyperbolic 3-manifold. Suppose that there is an infinite set $\mathcal{N}$ of primitive elements of $H_{2}(M ; \mathbb{Z})$ such that no element of $\mathcal{N}$ is represented by a (connected) fibroid. Then the maximal injectivity radius of $M$ exceeds 0.32798 .

Proof. If $\pi_{1}(M)$ has a non-abelian free quotient, then by [6, Theorem 1.3], the maximal injectivity radius of $M$ is at least $\frac{1}{2} \log 3=0.549 \ldots$ Now suppose that $\pi_{1}(M)$ has no non-abelian free quotient. If $\mathcal{N}$ is the set given by the hypothesis of Theorem 3, it now follows from [6, Proposition 2.1] that every element of $\mathcal{N}$ is represented by a connected incompressible surface, which by hypothesis cannot 
be a fibroid. Thus $\mathcal{N}$ has the properties stated in the hypothesis of Proposition 2 The latter result therefore implies that the maximal injectivity radius of $M$ exceeds 0.32798 .

If $M$ is a 3 -manifold whose first Betti number is at least 2 , then $H_{2}(M ; \mathbb{Z})$ has infinitely many primitive elements. If a non-trivial element of $H_{2}(M ; \mathbb{Z})$ is represented by a connected surface, it must be primitive, since it has intersection number 1 with a class in $H_{1}(M ; \mathbb{Z})$. Hence Theorem 3 implies:

Corollary 4. Let $M$ be a closed hyperbolic 3-manifold. Suppose that the first Betti number of $M$ is at least 2 and that $M$ contains no non-separating fibroid. Then the maximal injectivity radius of $M$ exceeds 0.32798 .

\section{REFERENCES}

[1] Ian Agol, Marc Culler, and Peter B. Shalen, Singular surfaces, mod 2 homology, and hyperbolic volume. I, arXiv:math.GT/0506396. To appear in Trans. Amer. Math. Soc.

[2] Ian Agol, Peter A. Storm, and William P. Thurston, Lower bounds on volumes of hyperbolic Haken 3-manifolds, J. Amer. Math. Soc. 20 (2007), no. 4, 1053-1077 (electronic). With an appendix by Nathan Dunfield. MR2328715 (2008i:53086)

[3] James W. Anderson, Richard D. Canary, Marc Culler, and Peter B. Shalen, Free Kleinian groups and volumes of hyperbolic 3-manifolds, J. Differential Geom. 43 (1996), no. 4, 738782. MR 1412683 (98c:57012)

[4] Marc Culler and Peter B. Shalen, Paradoxical decompositions, 2-generator Kleinian groups, and volumes of hyperbolic 3-manifolds, J. Amer. Math. Soc. 5 (1992), no. 2, 231-288. MR1135928 (93a:57017)

[5] _ Volumes of hyperbolic Haken manifolds. I, Invent. Math. 118 (1994), no. 2, 285-329. MR.1292114 (95g:57023)

[6] - Volumes of hyperbolic Haken manifolds. II, Proc. Amer. Math. Soc. 125 (1997), no. 10, 3059-3067. MR1422858 (97m:57022)

[7] Andrew Przeworski, Cones embedded in hyperbolic manifolds, J. Differential Geom. 58 (2001), no. 2, 219-232. MR1913942(2003e:57030)

Department of Mathematics, Statistics, and Computer Science (M/C 249), University of Illinois at Chicago, $851 \mathrm{~S}$. Morgan Street, Chichgo, Illinois 60607-7045

E-mail address: culler@math.uic.edu

Department of Mathematics, Statistics, and Computer Science (M/C 249), University

of Illinois at Chicago, 851 S. Morgan Street, Chicago, Illinois 60607-7045

E-mail address: shalen@math.uic.edu 